Lars H. Eriksen*

\title{
Die Polysemie in der Allgemeinsprache und in der juristischen Fachsprache Oder: Zur Terminologie der „Sache“ im Deutschen
}

\section{Einleitung ${ }^{\mathbf{1}}$}

Der folgende Beitrag erläutert das Verhältnis zwischen der juristischen Fachsprache und der Allgemeinsprache. Er besteht aus einer Darstellung der aktuellen Verhältnisse der Fachsprache des deutschen Rechts mit Beispielen. Da er als Einführung in die Problematik konzipiert wurde, sind hier auch didaktische und fremdsprachendidaktische Elemente für den Studierenden enthalten. Für den Übersetzer und Fachsprachenforscher dient der Beitrag als Grundlage für eine Diskussion und Vertiefung hinsichtlich der Sonderbedeutungen in der deutschen juristischen Fachsprache.

Die deutsche Rechtssprache ist gekennzeichnet durch ein eigenes Universum von Begriffen und Definitionen, welche sich von denjenigen der Allgemeinsprache unterscheiden. Für die Beschäftigung mit der Fachsprache sowohl in der Forschung als auch in der Praxis, beispielsweise in der Übersetzung, ist es nichtsdestotrotz erforderlich, sowohl die Allgemeinbedeutung als auch die juristische Sonderbedeutung der jeweiligen Begriffe zu kennen. So ist die Kenntnis der Rechts-Terminologie uner-

1 Der Beitrag beleuchtet einen Teilaspekt des vom Verfasser geleiteten Workshops „Die juristische Fachsprache“ anlässlich des AILA Fachsprachen-Kongresses „The 12. European Symposium on Language for Special Purposes" an der Freien Universität Bozen vom 30. August bis 3. September 1999 in Brixen (vgl. Eriksen/Luttermann im Druck).

* Dr. Lars H. Eriksen

Thyen, Theilen und Partner AG

Steuerberatungs- und Wirtschaftsprüfungsgesellschaft

Rathausplatz 15

D- 24937 Flensburg

E-mail:l.eriksen@ttup.com

Hermes, Journal of Linguistics no. 28-2002 
212

lässlich für eine Auseinandersetzung mit der Fachsprache des Rechts. Für die Einordnung eines Rechtstextes in seinen konkreten kommunikativen Zusammenhang ist die genaue Sachkenntnis von grundlegender Bedeutung. Der Übersetzer muss bei seiner Wiedergabe die entsprechende deutsche Bezeichnung für ein Wort aus einer fremden Rechtsordnung finden oder diese in einer fremden Sprache wiedergeben können.

\section{Zu der Begrifflichkeit: Was ist Polysemie ?}

Polysemie bedeutet Mehrdeutigkeit. Darunter wird verstanden, dass mehrere Bedeutungen in ein und demselben klanglichen (oder schriftlichen) Ausdruck enthalten sind. D.h., es ergeben sich - wie man es ausdrücken könnte - mehrere Bedeutungen für ein Wort.

Ein ganz anderer Blickwinkel ergibt sich aus der Bezeichnung Synonymie, welche den Fall der Eindeutigkeit zweier oder mehrerer Wörter beschreibt. Eine völlige Bedeutungsidentität ergibt sich dabei jedoch äußerst selten, da in den meisten Fällen dennoch stilistische Bedeutungsunterschiede bestehen. In der Regel beschränkt sich die Synonymie auf einen partiellen Aspekt der Bedeutung.

Die Mehrdeutigkeit besteht in den meisten Fällen darin, dass die Bedeutungen zweier oder mehrerer Wörter sich zwar in einem Bereich überlappen und in dieser Hinsicht als synonym zu bezeichnen, jedoch in anderen Bereichen nicht austauschbar sind. Gelegentlich wird hierfür auch die Bezeichnung „Begriffliche Überschneidung“ verwendet. ${ }^{2}$ Die jeweilige Teilbedeutung lässt sich in diesem Fall nur aus dem Zusammenhang erkennen. Beispielsweise kann das Wort Klage sowohl ein Streitgesuch an ein Gericht als auch ein allgemeinsprachlicher Ausdruck für die Äußerung des Schmerzes oder der Trauer sein. Dabei haben beide ihren Ursprung in dem Wort klaga mit seiner althochdeutschen Bedeutung Wehgeschrei.

\subsection{Andere Fälle von Mehrdeutigkeit}

Die Bezeichnung Homonymie ist in der Sprachwissenschaft der Oberbegriff für Homophonie und Homographie. Zur Homophonie zählt

\footnotetext{
${ }^{2}$ Vgl. Arntz/Picht 1989, S. 155 und 162.
} 
die Gruppe gleichklingender Wörter mit völlig anderer Bedeutung und mit unterschiedlicher Sprachgeschichte. Man denke hier beispielsweise an die Beispielpaare mehr oder Meer. ${ }^{3}$ Im Fall der gleichen Schreibung eines Wortes mit unterschiedlicher Bedeutung liegt dagegen Homographie vor. Nahezu als Schulbeispiel aus der Alltagssprache dient das Wort wei $\beta$, welches sowohl die Bedeutung „eine Farbe“ als auch die Bedeutung „ich wei $\beta^{\prime \prime}$ im Sinne eines Verbs besitzen kann. Im Folgenden werden jedoch ausschließlich Wörter aus dem Verhältnis der Alltagssprache zu der juristischen Fachsprache vorgestellt.

Beispielsweise lassen sich die unterschiedlichen Bedeutungen des Wortes Tenor im Sinne von entscheidender Spruch eines Gerichts oder ein Sänger mit hoher Stimmlage anführen, welche sich nicht durch ihre Schreibung, sondern nur durch ihre Betonung bei der Aussprache unterscheiden. Ein mehrdeutiges Wort wie z.B. das Gericht mit seinen alltagssprachlichen Bedeutungen wie Speise, Speisefolge oder Gerichtsgebäude sowie mit seinen fachsprachlichen Bedeutungen aus der Rechtssprache wie rechtsprechende Behörde, erkennendes Gericht (als Institution) oder Spruchkörper (Gremium) demonstriert, dass sich die Bezeichnungen nicht gegenseitig ausschließen, sondern sich alle drei vorgenannten Phänomene in einem Wort vereinen können. In diesem Fall liegt komplexe Mehrdeutigkeit vor, da das Wort sowohl als homophon und homograph als auch als polysem gelten kann. ${ }^{4}$

\section{Mehrdeutigkeit im Verhältnis von Fachsprache zu Allgemeinsprache}

Grundsätzlich kann mit v. Hahn das Verhältnis der Gemeinsprache zu einer Fachsprache nicht als ein Verhältnis der Vagheit im Sinne der Inexaktheit zur Präzision verstanden werden. ${ }^{5}$ Allerdings ist die Fachsprache des Rechts gerade durch besonders intensive Bemühung um sprachliche Exaktheit gekennzeichnet. Im Vergleich zum Sprachgebrauch in der Gemeinsprache fällt ihre exakte juristische Diktion auf. Nach meiner Auffassung sollte daher für die Fachsprache des Rechts eine Ausnahme gelten.

\footnotetext{
${ }^{3}$ Vgl. Arntz/Picht 1989, S. 135.

${ }^{4}$ Vgl. hierzu auch v. Hahn 1998, S. 379.

5 Vgl. v. Hahn 1998, S. 378.
} 


\section{4}

In der juristischen Fachsprache lassen sich jedoch auch Fälle einer „beabsichtigten Vagheit“ aufzeigen. Beispielsweise wurden aufgrund der Entwicklung des sozialen Zusammenlebens und des Rechts im Laufe der Zeit einige der Generalklauseln des BGB eingeführt, um Raum für rechtliche Interpretation zu ermöglichen. Hier wurden absichtlich starre und präzise Formulierungen vermieden. So ermöglicht z.B. § 242 BGB eine zeitgemäße Rechtsanwendung: „Der Schuldner ist verpflichtet, die Leistung so zu bewirken, wie Treu und Glauben mit Rücksicht auf die Verkehrssitte es erfordern." Wie beispielsweise aus einem verbreiteten Kommentar zum BGB zu entnehmen ist, enthält $\S 242$ BGB als sog. "offener Tatbestand" keinen Rechtssatz mit deskriptiven Tatbestandsmerkmalen. Der Inhalt dieser Bestimmung wird konkretisiert durch die Herausarbeitung von Funktionskreisen und Bildung von Fallgruppen. ${ }^{6}$ Eben aufgrund ihrer begrifflichen Vagheit wird diese Bestimmung allerdings bei einer juristischen Subsumtion seltener angewendet als allgemein in nicht-juristischen Kreisen angenommen wird.

Aufgrund der Existenz und der Verwendung sowohl der juristischen Fachsprache als auch der Alltagssprache in verschiedenen Bereichen des Lebens ergeben sich unterschiedliche Bedeutungen gleichgeschriebener Wörter.

Eine Fachsprache wird daher im Folgenden als eine Sondersprache der fachlichen Spezialisierung verstanden, die durch Besonderheiten beispielsweise hinsichtlich der Lexik, der Semantik, der Wortbildung oder der Syntax geprägt ist. Zur Fachsprache gehört jedoch notwendigerweise auch die soziale Komponente aufgrund ihres Gebrauches in einer Gemeinschaft - denn letztlich ist der Fachdiskurs mit Fachkollegen Anlass und Ziel und damit maßgeblich für das Vorliegen einer fachsprachlichen Kommunikation. Demgegenüber kann sich die Verwendung einer Fachsprache im nicht-fachlichen Rahmen als nicht-adäquat für eine Kommunikation erweisen. Gegenüber einem Nicht-Fachmann, einem Laien, erfordert die Situation hier die Umsetzung der fachsprachlichen Inhalte in allgemeinverständliche Terminologien durch die Benutzung der Allgemeinsprache.

\footnotetext{
${ }^{6}$ Vgl. Palandt § 242 , Rn. 2.
} 
Die Allgemeinsprache, welche bekanntlich auch als Alltagssprache, Umgangssprache, Gemeinsprache oder schlicht als gewöhnliche Sprache bezeichnet wird, ist die Sprache des täglichen Lebens. ${ }^{7}$ Sie steht im Gegensatz sowohl zur Hochsprache als auch zur Schriftsprache. Die Allgemeinsprache ist durch hohe Akzeptanz, weite Verbreitung im Sprachgebiet sowie durch nicht wesentliche Besonderheiten in dialektaler, sozialer, fachlicher oder altersmäßiger Hinsicht gekennzeichnet. ${ }^{8}$ Es handelt sich um die Sprache, die von jedermann im Laufe eines (All-)Tages verwendet wird.

\subsection{Die Polysemie im Verhältnis der juristischen Fachsprache zur Allgemeinsprache}

Viele Ausdrücke der Allgemeinsprache und aus dem Fachbereich des Rechts besitzen eine scheinbar - aber nur scheinbar - identische Terminologie. Nachfolgend werden Beispiele für diese Mehrdeutigkeit anhand von Fällen aus der deutschen juristischen Sprache aufgezeigt und den entsprechenden Bedeutungen der Allgemeinsprache gegenübergestellt. Dabei bezieht sich der folgende Vergleich auf Beispiele aus dem Bürgerlichen Recht des Rechtssystems der Bundesrepublik Deutschland. Ich beschränke mich, wie bereits eingangs erwähnt, deshalb zunächst auf den zentralen Bereich des Zivilrechts.

\subsubsection{Die Sache im Zivilrecht}

Im Bereich des Zivilrechts ist eine Sache gem. § 90 Absatz 1 BGB, dem wohl wichtigsten deutschen Gesetz, ,ein körperlicher Gegenstand". Dementsprechend rechnet der Jurist zu Sachen Festes, Luftförmiges sowie Flüssiges. Diese spezifische Bedeutung im juristischen Sprachgebrauch beruht auf der juristischen Fachsprache des 19. Jahrhunderts. Das BGB trat nach etwa 30-jähriger Vorarbeit am 1.1.1900 in Kraft. Die Wortwahl des BGB lässt sich daher als wohlüberlegt bezeichnen. Bereits damals stand der fachspezifische Wortgebrauch des Bürgerlichen Gesetzbuches im Gegensatz zu demjenigen der Allgemeinsprache. In

\footnotetext{
${ }^{7}$ Vgl. Eichhoff 1977, Bd. I, S. 5f sowie Küpper 1988 passim.

${ }^{8}$ Vgl. Eriksen 1999, S. 38f.
} 
216

der Umgangssprache bezeichnet Sache etwas Festes oder dem Begriff nach etwas, was man sich im Allgemeinen als fest vorstellt. Die Alltagssprache meint mit Sachen verallgemeinernd , nicht näher bezeichnete Gegenstände“ oder einen ,nicht näher bezeichneten Vorgang“ (vgl. Duden, Bd. V, 1980). Luftförmige Gegenstände wie Gase oder Dämpfe werden dabei nicht als Sache empfunden. Auch Flüssigkeiten wie z.B. Wasser oder Farben werden im Allgemeinen nicht als Sachen bezeichnet. Der Wahrig, ein anerkanntes aktuelles deutsches Wörterbuch, welches die heutige Verwendung in der Alltagssprache gesondert verzeichnet, belegt diese Auffassung: 9

„Sache (n):

1. ein Gegenstand, Ding;

2. (Rechtswesen) jeder körperliche Gegenstand;

3. Angelegenheit;

4. Frage;

5. Fall;

6. Aufgabe, Pflicht;

7. Unternehmen, Begebenheit"“

Der Jurist wählt bei der Auslegung von Gesetzen häufig die Allgemeinsprache zum Ausgangspunkt zur Konkretisierung von Fachbegriffen. So nimmt er beispielsweise im Bereich des Zivilrechts zur Umschreibung des Fachterminus Sache die Verkehrssitte zum Maßstab. D.h., es wird die im alltäglichen Verkehr allgemein übliche Auffassung breiter Kreise der Bevölkerung zum Ausgangspunkt genommen. ${ }^{10}$ Es wird dabei nach juristischen Verständnis keine rechtliche Wertung vorgenommen, sondern eine Sache zuerst nach sogenannten allgemeinen Anschauungen und Vorstellungen der Bevölkerung beurteilt. Erst in einem zweiten Schritt erfolgt die juristische Wertung. Es kommt demnach nicht auf den letzten Stand der physikalischen Wissenschaft an. ${ }^{11}$ So begründet der Jurist konsequent und gemäß den Grundsätzen des $\S 90$ BGB nachvollziehbar,

\footnotetext{
${ }^{9}$ Vgl. Wahrig, 1997, Nummerierung durch den Verfasser.

10 Es handelt sich wie bereits vom Reichsgerichtshof festgestellt wurde, um eine der grundlegenden Auffassungen im deutschen Recht, vgl. Entscheidungen des Reichsgerichtshofes Band 169 Seite 125.

${ }^{11}$ Vgl. Palandt / Heinrichs Rn. 2 zu $§ 90$ BGB.
} 
dass Licht und Strom keine Sachen im Sinne des Gesetzes sein können. ${ }^{12}$ Andererseits können nach der Rechtsprechung der letzten Jahre auch Datenträger - d.h. eine Diskette, eine CD-ROM oder eine Festplatte eine Sache sein. ${ }^{13}$ Allerdings kann Software nach juristischem Grundsatz nicht als ein körperlicher Gegenstand gesehen werden. Denn Computerprogramme (Software) bestehen aus binären Zahlenpaaren in einer bestimmten Kodierung, die in elektronischer Form auf einem Datenträger aufbewahrt werden. Das Oberlandesgericht Stuttgart vertrat und begründete aber im Jahre 1989 ebenso mit der Verkehrsauffassung die gegenteilige Auffassung, Software sei demnach eine Sache. ${ }^{14}$ Diese Auffassung ist seitdem gängig im Zivilrecht. Mit dem Beispiel Software steht der juristische Sprachgebrauch im Zivilrecht auch im Gegensatz zur Allgemeinsprache, die Software, also Computerprogramme, wohl kaum als Sache anerkennt.

Auch Luft an sich wird nicht als Sache im zivilrechtlichen Sinn gesehen. Erst wenn die Luft ihren Zustand als allgemeinzugängliches Gut verliert und beispielsweise als komprimierte Luft gehandelt werden kann, wird sie u.U. im juristischen Sinne als juristische Sache gesehen. Sollte gute und saubere Luft Mangelware werden und ließe sich diese beispielsweise als Ozondusche oder Sauerstoffbad verkaufen, könnte damit allerdings auch im Sprachgebrauch der Allgemeinsprache das freie Gut Luft zu einer Sache werden.

\subsubsection{Das Tier als Sache}

Der juristische Sprachgebrauch erkennt ganz im Gegensatz zum Sprachgebrauch der Allgemeinsprache Tiere als Sachen an. Hier proklamiert der im Jahre 1990 aufgrund des „Gesetzes zur Verbesserung der Rechtsstellung des Tieres“ neu eingefügte $\S$ 90a, Satz 1 BGB: „Tiere sind keine Sachen". Diese Formulierung wurde im Wesentlichen als Folge der Proteste von Tierschützern in das BGB aufgenommen. Man beabsichtigte, den Grundgedanken, dass das Tier als Mitgeschöpf nicht

\footnotetext{
12 Vgl. Entscheidungen des Reichsgerichtshofes in Zivilsachen, Band 86, S. 14, und Münchener Kommentar zum BGB / Holch Rn. 20 zu § 90 BGB.

${ }^{13}$ Vgl. Entscheidungen des Bundesgerichtshofes in Zivilsachen, Band 102, S. 144 und Band 109, S. 100.

${ }^{14}$ Vgl. Neue Juristische Wochenschrift 1989, S. 2635.
} 


\section{8}

der Sache gleichgestellt werden darf, in den Vordergrund zu stellen. Allerdings werden trotz dieser Umformulierung des Wortlautes des Gesetzes im heutigen juristischen Sprachgebrauch Tiere weiterhin als Sachen gesehen. Ja, selbst ein allgemein anerkannter juristischer Kommentar zum BGB gibt zum Ausdruck: „Im Ergebnis ist § 90a BGB eine gefühlige Deklamation ohne wirklichen rechtlichen Inhalt. "15 Damit befindet sich aber die Rechtslehre und Rechtspraxis im Widerspruch zum deklarierten Zweck des $\S 90$ a BGB.

\subsubsection{Vertretbare und verbrauchbare Sachen}

Des Weiteren gliedert die moderne Rechtssprache Sachen nach dem Gesichtspunkt der Vertretbarkeit und dem Gesichtspunkt des Verbrauchs (vgl. $\S \S 91$ und 92 BGB).

Vertretbare Sachen sind Sachen, ,die im Verkehre nach Zahl, Maß oder Gewicht bestimmt zu werden pflegen." (vgl. § 91 BGB). Es wird darunter eine Sache verstanden, die ,sich von anderen der gleichen Art nicht durch ausprägte Individualisierungsmerkmale abhebt und daher ohne weiteres austauschbar ist."16

Dabei geht die Jurisprudenz in der Sprache eigene Wege. So kann auch eine Flüssigkeit wie z.B. Wein als eine vertretbare Sache angesehen werden, wenn es sich um eine Gattung handelt, welche sich durch Rebsorte, Lage und Qualitätsstufe kennzeichnet (Palandt ebd.). Als vertretbare Sachen im juristischen Sinn werden daher u.a. auch Massenfabrikate moderner Konsumgüter wie Kraftfahrzeuge, Rundfunkgeräte oder Rechner aufgefasst. Aber eine Sache kann sich auch als nicht vertretbar erweisen. Dabei bewegt sich der juristische Sprachgebrauch auf völlig anderem Wege - weg von der Alltagssprache. Denn unter nicht vertretbaren Sachen werden beispielsweise Werkstücke verstanden, die wegen ihrer Eigenart nicht ersetzt werden können. Es handelt sich um unersetzbare Gegenstände, die als Einzelstück durch einen Handwerker oder Künstler hergestellt wurden.

${ }^{15}$ Vgl. Palandt / Heinrichs Rn. 1 zu $§ 90 a$ BGB).

16 Vgl. Palandt / Heinrichs Rn. 1 zu $§ 91$ BGB. 
Dem Wort vertretbar kommt in der Rechtssprache eine besondere Bedeutung zu. Verstanden wird darunter das Ergebnis einer bestimmten Handlung, die nur eine bestimmte Person vollziehen kann. Eine besonders schwierige Übersetzung beispielsweise kann in diesem Sinne als eine sogenannte nicht vertretbare Handlung bezeichnet werden, die nicht jeder Übersetzer zu vollziehen vermag, sondern nur ein bestimmter besonders dazu befähigter.

Verbrauchbare Sachen sind „bewegliche Sachen, deren bestimmungsmäßiger Gebrauch in dem Verbrauch oder der Veräußerung besteht.“ (vgl. § 91, Absatz 1. BGB). Der Jurist etabliert dadurch eine genaue Kategorisierung des Begriffsfelds Sache, die der Alltagssprache in dieser Genauigkeit unbekannt ist. Damit befindet sich die Rechtssprache im Gegensatz zur Allgemeinsprache: Eine derart genaue Kategorisierung wird von der Allgemeinsprache so nicht mitgetragen.

\subsubsection{Die Fachpolysemie: Das Wort Sache in anderen Rechtsgebieten}

Ein Verhältnis, welches als Polysemie bezeichnet werden kann, besteht nicht nur zwischen der Alltagssprache und einer Fachsprache. In einer Fachsprache kann sich für ein Wort in verschiedenen Fachbereichen eine Polysemie ergeben. Arntz und Picht geben an, die Polysemie trete häufiger in der Gemeinsprache als in der Fachsprache auf. ${ }^{17}$ Jedoch kann diese Annahme in ihrer Allgemeinheit wohl nicht für die juristische Fachsprache gelten, denn die umfangreiche juristische Fachsprache bietet mehrere Beispiele für eine sog. Fachpolysemie. Es wird in der juristischen Fachsprache allerdings nicht versucht, wie sonst häufig in anderen Fachsprachen, eine Normung einzuführen. ${ }^{18}$ Insofern müssen sich Sprachwissenschaftler bei der Untersuchung von Rechtstexten Rechtskenntnisse aneignen, wenn sie die Fachsprache des Rechts verstehen wollen. Im deutschen Recht wird beispielsweise das Wort Sache in verschiedenen Rechtsgebieten unterschiedlich verwendet. Die Subsprachen - u.a. des Zivilrechts, des Strafrechts, des Prozessrechts, des Verwaltungsrechts sowie die deutsche Terminologie des Europarechts - inter-

\footnotetext{
17 Vgl. Arntz/Picht 1989, S. 135.

18 Vgl. Fluck 1996, S. 47f.
} 
pretieren unterschiedliche Bedeutungen hinein, die jedoch weitestgehend den zivilistischen Begriff des $\S 90$ BGB zum Ausgangspunkt haben.

Wie erwähnt, wird der Begriff Sache als ,körperlicher Gegenstand“ im BGB mit Wirkung für alle Rechtsgebiete grundlegend definiert. Im Bereich des Verwaltungsrechts gilt das Kriterium Körperlichkeit aber nicht immer als Definitionsmerkmal für eine Sache. Beispielsweise wird „,der Luftraum über dem Straßenkörper" u.a. gem. § 1, Absatz 4, Nr. 2 Bundesfernstraßengesetz oder ,der Luftraum“ gem. § 1 Luftverkehrsgesetz als eine sogenannte ,"̈ffentliche Sache“ gesehen. Diese andere gegenständliche Auffassung von gewöhnlicher Luft im öffentlichen Recht steht im Gegensatz zur Begriffsbildung im Zivilrecht. Ebenso wird von „öffentlichen Sachen“i.S. „res publica“ gesprochen, wenn die Rede von Sachen im öffentlichen Eigentum ist, wie beispielsweise vom Gebäude einer Schule oder eines Polizeistreifenfahrzeugs.

Der spezifische Sprachgebrauch des Strafgesetzbuches geht wiederum vom Gegenstandsbegriff des Zivilrechts aus. Allerdings verlässt das Strafrecht den Boden der gemeinsamen Definition - auch nach der Einführung des $\S 90 \mathrm{a}$ BGB - , wenn es z.B. die Tötung eines Hundes als Sachbeschädigung ansieht. ${ }^{19}$ Im Gegensatz zur Auffassung im Zivilrecht (s.o.) wird Software im Strafrecht nicht als Sache aufgefasst. Die Anwendung des Begriffes Sache im Strafrecht beruht auf einer anderen Definition, wie bereits das Reichsgericht feststellte: ,Der strafrechtliche Begriff Sache ist als selbständiger, vom Zivilrecht unabhängiger öffentlich-rechtlicher Begriff nur dem Strafrecht selbst zu entnehmen. " 20 Der Gedankengang des $\S 90$ a Satz 1 BGB dient zwar als Ausgangspunkt für die Definition der Sache im Strafrecht, er wird jedoch nicht als maßgeblich für diese Subsprache der Fachsprache des Rechts angesehen.

Als weiteres Beispiel für die unterschiedlichen Bedeutungen in der Fachsprache des Rechts kann im Zivilprozessrecht auch die Rede sein von einer „Rechtssache“ oder "Sache“ im Sinne von „Verfahren“. So ist der erste Satz des Richters in vielen Zivilprozessen: „Zum Aufruf kommt die Sache A gegen B. " Diese Verwendung stellt sprachgeschicht-

${ }^{19}$ Vgl. Dreher/Tröndle Rn. 2 zu § 242 StGB.

${ }^{20}$ Vgl. Entscheidungen des Reichsgerichts in Strafsachen, Band 32, S. 179. 
lich gesehen die ursprüngliche Bedeutung als ,,Streitigkeit “oder „Zwist “ in der Bedeutung von ,vor dem Richter ausgetragene Streitigkeit" dar.

Die Sachkunde, die dem Sachverständigen oder dem sachkundigen Zeugen bei Gericht abverlangt wird, bezeichnet ebenfalls etwas anderes als nur Kenntnis von einem gegenständlichen Vorgang. Es kann ihm auch Sachkenntnis von einem Vorgehen oder einer Methode, und damit von einem nicht körperlichen Gegenstand, abverlangt werden.

Im Europarecht hat sich der Sprachgebrauch am französischen Muster orientiert. Demnach verwendet die offizielle Aktenordnung des Europäischen Gerichtshofes in der deutschen Fassung die Bezeichnung „Rechtssache“, abgekürzt „Rs.“. An entsprechender Stelle wird in der Registerverfügung der deutschen Gerichte das Aktenzeichen „O" für „allgemeine Zivilsachen erster Instanz beim Landgericht" oder „Ds“ für „Strafverfahren vor dem Einzelrichter am Amtsgericht“ verwendet. Insofern ergibt sich damit in der Fachsprache des Rechts eine weitere unterschiedliche Bedeutung des Wortes Sache.

\section{Fazit: Die Sache mit der Sache}

Wie aus den wenigen Beispielen zu erkennen war, ergibt sich eine Vielfalt von Bedeutungen nicht nur im Verhältnis zwischen Fachsprache und Allgemeinsprache, sondern auch hinsichtlich der sogenannten Fachpolysemie d.h. im Verhältnis der Fachsprache zu mehreren ihrer Subsprachen. Kenntnisse der Polysemie zwischen der Fachsprache und der Allgemeinsprache sowie der Fachpolysemie sind für die Arbeit des Übersetzers und Dolmetschers von entscheidender Bedeutung. Dies erfordert sowohl Kenntnis der verschiedenen detaillierten Bedeutungen in der juristischen Fachsprache als auch in der Allgemeinsprache.

\section{Schrifttum}

Arntz, Reiner \& Picht, Heribert (1989). Einführung in die Terminologiearbeit. Hildesheim / Zürich / New York: Olms.

Daum, Ulrich (1998): Fingerzeige für die Gesetzes- und Amtssprache. Rechtssprache bürgernah. 11. Aufl., Wiesbaden: Quelle \& Meyer.

de Saussure, Ferdinand (1967). Grundfragen der allgemeinen Sprachwissenschaft. Berlin: de Gruyter.

Dreher, Eduard \& Tröndle, Herbert (1999). Strafgesetzbuch und Nebengesetze. 49. Aufl., München: Beck. 
222

Duden (1980). Das große Wörterbuch in sechs Bänden. Mannheim / Wien / Zürich: Duden.

Eichhoff, Jürgen (1977). Wortatlas der deutschen Umgangssprachen. Bd. I - III. Bern, München, New Providence, London, Paris: Saur.

Eriksen, Lars (1995). Die Fachsprache des Juristen. In IDV-Rundbrief. Zeitschrift des Internationalen Deutschlehrerverbandes 54. 30 - 37.

Eriksen, Lars (1999). Idiome und Phrasen aus der aktuellen deutschen Umgangssprache. Teil I: Aus dem aktuellen Wortschatz des Positiven. In IDV-Rundbrief. Zeitschrift des Internationalen Deutschlehrerverbandes 63.36-42.

Eriksen, Lars (2001). Die Mittlerfunktion der juristischen Fachsprache. In: Mayer, Felix (ed.): Language for Special Purposes: Perspectives for the New Millennium. Tübingen: Narr. 603-612.

Eriksen, Lars \& Luttermann, Karin (im Druck). Die Fachsprache des Rechts. Münster: LIT.

Fluck, Hans-Rüdiger (1991). Fachsprachen. Tübingen: Francke.

v. Hahn, Walther (1998). Vagheit bei der Verwendung von Fachsprachen. In: Hoffmann, Lothar et al. (Hrsg.) (1998). Fachsprachen. 1. Halbband. Berlin / New York: de Gruyter. 378-382.

Küpper, Heinz (1988). Illustriertes Lexikon der deutschen Umgangssprache. Bd. I VIII. Stuttgart: Klett.

Münchener Kommentar zum Bürgerlichen Gesetzbuch (1995). Bd. 1., Allgemeiner Teil, 3. Aufl. München: Beck.

Palandt, Otto (1999). Kommentar zum Bürgerlichen Gesetzbuch. 58. Aufl. München: Beck.

Rehbein, Jürgen (1998). Die Verwendung von Institutionensprache in Ämtern und Behörden. In: Hoffmann, Lothar et al. (Hrsg.) (1998). Fachsprachen. 1. Halbband. Berlin / New York: de Gruyter. 666 - 675.

Wahrig (1997). Deutsches Wörterbuch. Gütersloh: Bertelsmann. 